Ann. Biol. anim. Bioch. Biophys., I965, 5 (I), I 45 -I 49.

\title{
ACTION DES HORMONES GONADOTROPES SUR LE TESTICULE DE L'AGNEAU
}

\author{
M. COUROT \\ avec la collaboration technique de Claudine Pisselet et Christiane Rrchetris \\ Laboratoire de Physiologie de la Reproduction, \\ Centre national de Recherches zootechniques, \\ Jouy-en-Josas (Seine-et-Oise)
}

\section{SOMMAIRE}

Cinquante-trois agneaux dont les testicules ne manifestaient encore aucune activité spermatogénétique, ont reçu deux fois par jour pendant 5 ou I 5 jours des produits gonadotropes du commerce en injection sous-cutanée (poudre antehypophysaire, FSH, ICSH). Ils ont été abattus une demijournée après la dernière injection.

Dans ces conditions, on observe une augmentation du nombre des cellules de sontien, précurseurs des cellules de Sertoli, avec le traitement FSH dès 5 jours et arec tous les produits après 15 jours d'expérience.

Il n'y a pas d'augmentation du nombre des cellules germinales primordiales, mais on observe la formation précoce de spermatocytes de premier ordre chez un certain nombre d'animaux avec chacun des produits employés.

\section{INTRODUCTION}

Dans une expérience préliminaire, nous avons montré qu'il était possible d'obtenir, chez l'Agneau, une stimulation importante des cellules de soutien, précurseurs des cellules de Sertoli, sans réponse des cellules germinales primordiales à la suite d'un traitement de courte durée (5 jours) par des hormones gonadotropes (CouroT, I962). Nous avons cherché dans ce travail à savoir si une intervention prolongée (I5 jours) permettait de mettre en évidence une action sur les cellules germinales.

\section{MATÉRIEL ET MÉTHODES}

Cinquante-trois agneaux de race $I l e-d e-F r a n c e$ de 15 ì $2 \mathrm{I} \mathrm{kg}$ ont reçu différents produits gonadotropes - poudre acétoni (ue antéhypophysaire ovine brute $\left(0,75^{-1,5}\right.$ et $\left.3 \mathrm{~g} / \mathrm{j}\right)$, FSH ou ICSH "Armour " (2,8-5,6 et $\mathrm{x} \mathrm{I}, 2 \mathrm{U}$ Armour $j \mathrm{j})$ - - en injection sous-cutanée matin et soir pendant 5 ou 
I 5 jours. Les testicules ont été prélevés par castration I 2 à I 5 heures après la dernière injection, pesés puis fixés dans le mélange de Bouin-Iollande additionné de ro p. ioo d'une solution saturée de sublimé.

Les techniques histologiques mises en veuvre furent comparables à celles utilisées précédemment. Les résultats ont été exprimés en nombres totaux de cellules par testicule, calculés suivant la formule :

$$
\mathrm{N}=\frac{n \cdot \mathrm{CV}^{\circ},(\mathrm{P}-p)}{\pi \mathrm{D}^{2} / 4 \cdot \mathrm{E}}=\frac{n \cdot \mathrm{I}}{\mathrm{E}}
$$

où n est le nombre moyen de cellules d'une catégorie donnée présent par section transversale de cordon sexuel de diamètre $\mathrm{D}, \mathrm{C}$ le coefficient de contraction après fixation (ici $\mathrm{C}=0,65$ ), $\mathrm{V} \%$ le volume relatif des tubes séminifères, $\mathrm{P}$ le poids frais du testicule, $p$ celui de l'albuginée (1) et $\mathbf{E}$ l'épaisseur de la coupe; $L$ est la longueur totale des cordons sexuels.

\section{RÉSULTATS}

Les résultats obtenus sont présentés dans les tableaux I et 2. L'ensemble des traitements appliqués provoquent une augmentation nette du poids des testicules. L'injection de poudre antehypophysaire ou de FSH semble la plus efficace, mais il n'y a pas d'augmentation de réponse arec la dose administrée.

Le diamètre des cordons sexuels s'accroît sous l'effet de la poudre antehypophysaire et de l'hormone folliculo-stimulante alors qu'il ne varie pas après l'administration d'hormone interstitielle-stimulante. La longueur des cordons sexuels augmente avec tous les traitements, surtout lorsqu'ils sont de longue durée.

Étant donné que le volume relatif occupé par les cordons sexuels n'est modifié par aucun des traitements, il faut admettre que ceux-ci ont stimulé aussi le tissu interstitiel.

Sur le plan cellulaire, on observe une augmentation du nombre total des cellules de soutien dans le testicule. Ceci est déjà significatif avec le traitement court de FSH et le devient avec tous les produits essayés (y compris ICSH) en traitement long. Il y a donc dans les produits étudiés un ou plusieurs facteurs qui stimulent la multiplication des cellules de soutien dans les cordons sexuels. Les noyaux de ces cellules ont une allure normale et quelques-uns sont en division. Parfois, en cas de stimulation très intense, ils présentent des figures particulièrement étirées comme celles signalées dans une note précédente (Cocrot, rg62). Cependant, nous n'avons pas observé, même avec des traitements longs, de différenciation norphologique de cellules de soutien en cellules de Sertoli typiques.

En ce qui concerne les cellules germinales, s'il n'y a pas d'effet significatif des produits gonadotropes employés sur le nombre total des cellules germinales primordiales, on observe cependant un phénomène qualitatif extrêmement important: chez un certain nombre d'animaux, il y a eu production précoce de quelques spermatocytes de premier ordre (stade zygotène). Ceci s'observe surtout chez des animaux soumis au traitement de longue durée avec tous les produits essayés. Jamais nous ne l'observons chez les animaux témoins. Il faut toutefois souligner que dans les

(1) Celui-ci a été calculé à partir des relations linéaires que nous avons établies entre le poids testiculaire et le poids de l'albuginée lors du développement des testicules de l'Agneau (Covkot, non publié)

$$
\begin{aligned}
& \mathrm{P}<20 \mathrm{H} \longrightarrow p=0,1 \mathrm{I}+\mathrm{P}+0,027 \\
& \mathrm{P}^{\prime}>20 \mathrm{H} \rightarrow \mathrm{p}=0,05+\mathrm{P}+2,95
\end{aligned}
$$


HORMONES GONADOTROPES CHEZ L'AGNEAU

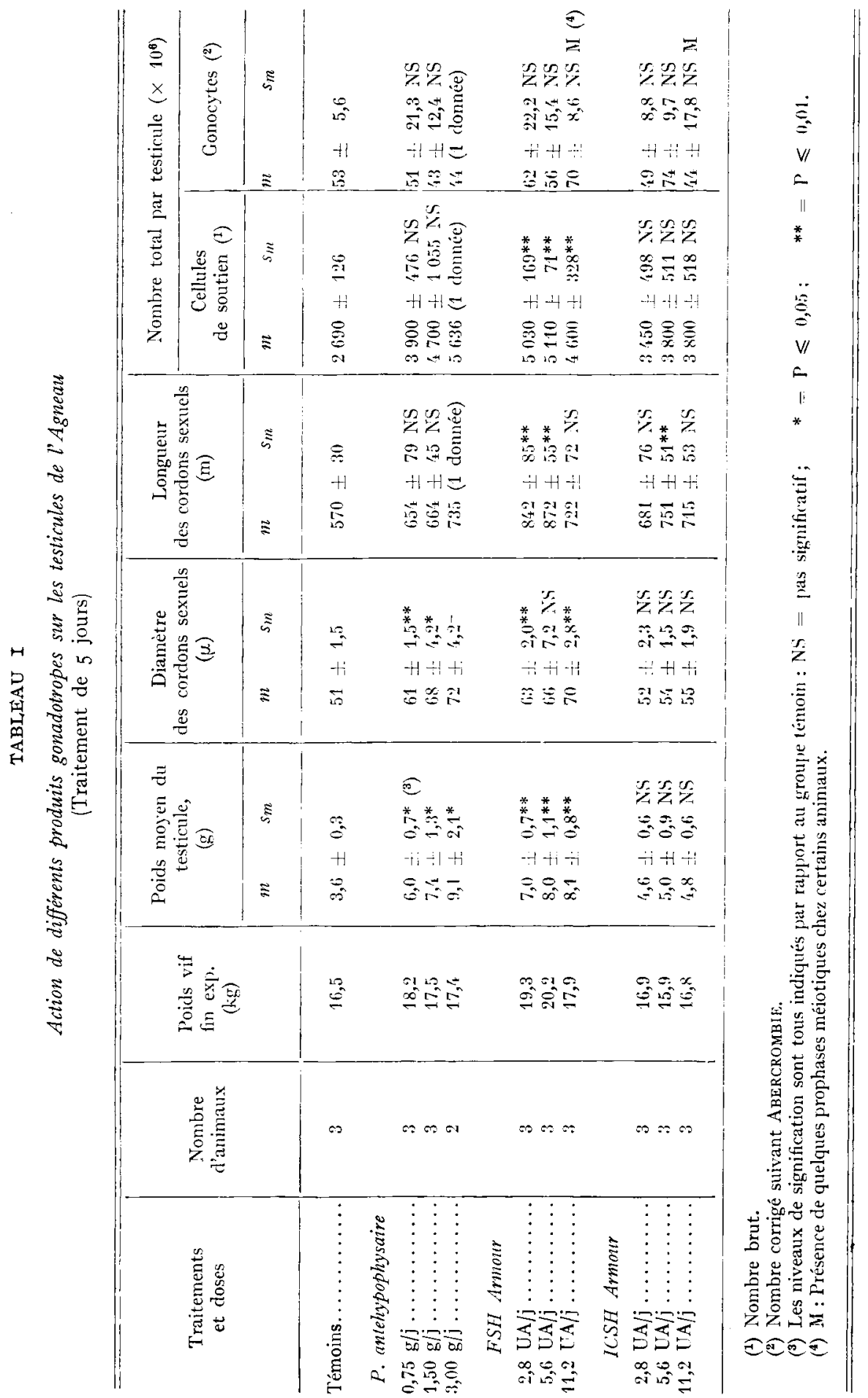




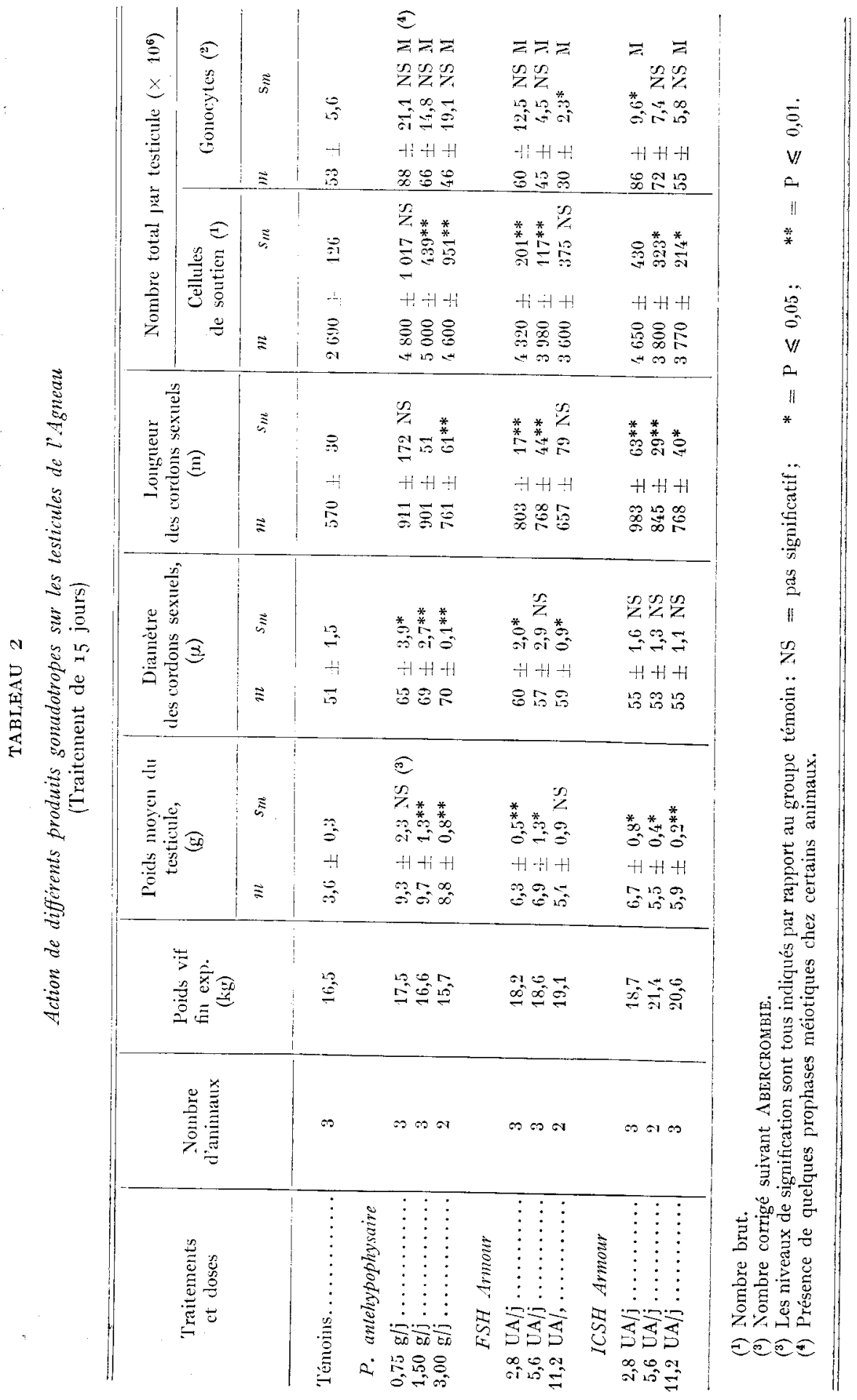


conditions de notre expérience, il ne se forme que très peu de spermatocytes primaires. Ce résultat montre que s'il est impossible d'accélérer la spermatogenèse lorsqu'elle est active, (cf. revue sur ce sujet par ORTAVANT et CoUROT, Ig64), on peut en avancer la mise en route avec un traitement approprié.

\section{CONCLUSION}

Chez l'agneau dont les testicules ne manifestent encore aucune activité spermatogénétique, l'injection prolongée d'hormones gonadotropes agit sur les cordons sexuels. Nous avons pu confirmer l'action de ces produits sur les cellules de la lignée sertolienne dont le nombre a été augmenté. En outre, bien qu'il n'y ait pas eu d'augmentation du nombre total des cellules germinales primordiales, il a été possible de provoquer une différenciation précoce de la spermatogenèse, ce qui n'avait pas été obtenu jusqu'à présent chez les mammifères.

Il importe maintenant de préciser le rôle respectif éventuel des hormones exogènes et endogènes dans ces phénomènes.

Reçu pour publication en novembre 1964 .

\section{SUMMARY}

\section{ACTION OF GONADOTROPIC IORMONES ON THE TESTIS OF THE PREPUBERAL LAMB}

Fifty-three Ile-de-France lambs, whose testes were not yet showing any spermatogenic activity, were injected twice a day for 5 or I 5 consecutive days with different gonadotropic hormones (sheep antepituitary extract, Armour FSH and Armour ICSHI). The testes were removed I2-I 5 hours after the final injection and were weighed and prepared for a quantitative histological investigation.

Under the conditions of the experiment the products injected (with the exception of ICSH, 5 days) induced an increase in test is weight. The diameter of the sex cords was increased after injection with antepituitary extract or with FSH. An increase in the number of the precurcors of the Sertoli cells was observed as a result of injecting with FSI for 5 days and with all the products for I 5 days. There was no increase in the number of primordial germ cells but the formation of primary spermatocytes was observed in a certain number of animals with each of the products emploved.

\section{RÉFÉRENCES BIBLIOGRAPHIQUES}

Courot M., 1962. Action des hormones gonadotropes sur le testicule de lagneau impubere. Réponse particulière de la lignée sertolienne. Amn. Biol. anim. Bioch. Biophys., 2, 15\%-162.

Ortavant R., Courot M., Ig64. I'roblèmes concernant l'action des hormones gamétocinétiques sur la spermatogenèse des mammifères. Arch. Biol. (Liège), 75, 625-667. 